\title{
Thermodynamics of Heat-Shock Response
}

\author{
Kristine Bourke Arnvig, ${ }^{1}$ Steen Pedersen, ${ }^{1}$ and Kim Sneppen ${ }^{2}$ \\ ${ }^{1}$ Institute of Molecular Biology, Øster Farimagsgade 2A, 1353 Copenhagen K, Denmark \\ ${ }^{2}$ Nordita, Blegdamsvej 17, 2100 Copenhagen Ø, Denmark
}

(Received 29 July 1999)

\begin{abstract}
Production of heat-shock proteins is induced when a living cell is exposed to a rise in temperature. The heat-shock response of protein DnaK synthesis in E.coli for temperature shifts $T \rightarrow T+\Delta T$ and $T \rightarrow T-\Delta T$ is measured as a function of the initial temperature $T$. We observe a reversed heat shock at low $T$. The magnitude of the shock increases when one increases the distance to the temperature $T_{0} \approx$ $23{ }^{\circ} \mathrm{C}$, thereby mimicking the nonmonotonous stability of proteins at low temperature. This suggests that stability related to hot as well as cold unfolding of proteins is directly implemented in the biological control of protein folding.
\end{abstract}

PACS numbers: 87.14.Ee, 36.20.-r, 82.60.-s, 87.16.-b

Chaperones direct protein folding in the living cell by binding to unfolded or misfolded proteins. The expression level of many of these catalysts of protein folding changes in response to environmental changes. In particular, when any living cell is exposed to a temperature shock the production of these evolutionary conserved proteins is transiently increased [1]. The heat-shock (HS) response in E.coli involves about 40 widely dispersed genes and is mediated through the $\sigma^{32}$ protein [2,3]. The $\sigma^{32}$ binds to RNA polymerase (RNAp), where it displaces the $\sigma^{70}$ subunit and thereby changes RNAp's affinity to a number of promoters. This induces production of the heat-shock proteins. If the gene for $\sigma^{32}$ is removed from the E.coli genome, the HS is suppressed $[2,4]$ and also the cell cannot grow above $20^{\circ} \mathrm{C}$.

The HS is fast. In some cases it can be detected by a changed synthesis rate of, e.g., the chaperone protein DnaK, about a minute after the temperature shift. Given that the DnaK protein in itself takes about 45 seconds to synthesize, the observed fast change in DnaK production must be very close to the physical mechanism that triggers the response. We will argue for a mechanism that does not demand an additional synthesis of $\sigma^{32}$ and thus postulate that a changed synthesis of $\sigma^{32}$ only plays a role in the latter stages of the HS. To quantify the physical mechanism we measure the dependence of HS with initial temperature and find that the magnitude of the shock is inversely proportional to the folding stability of a typical globular protein.

This paper measures the expression of protein DnaK. Steady state levels can be found in [5,6]; they vary from approximately 4000 at $T=13.5$ to approximately 6000 at $37^{\circ} \mathrm{C}$. DnaK is a chaperone and it has a high affinity for hydrophobic residues [7]; as these signal a possible misfold, $\sigma^{32}$ controls the expression of DnaK by binding to the RNAp. One expects at most a few hundred $\sigma^{32}$ in the cell, a number which is dynamical adjustable because of the short in vivo half-life of $\sigma^{32}\left(0.7 \mathrm{~min}\right.$ at $42{ }^{\circ} \mathrm{C}$ and $15 \mathrm{~min}$ at $\left.22^{\circ} \mathrm{C}[8,9]\right)$. The lifetime $\sigma^{32}$ is known to increase transiently under the HS.
The measurement was on an E.coli $\mathrm{K} 12$ strain grown on an $A+B$ medium with a ${ }^{3} \mathrm{H}$ labeled amino acid as described in [10]. After the temperature shift we extracted samples of the culture at subsequent times. Each sample was exposed to radioactive labeled methionine for $30 \mathrm{sec}-$ onds, after which $10^{5}$-fold nonradioactive methionine was added. Methionine absorbs very rapidly, and is then used in protein synthesis. Protein DnaK was separated by 2-dimensional gel electrophoresis, and the amount of synthesis during the 30 seconds of labeled methionine exposure was determined first with respect to ${ }^{3} \mathrm{H}$ counts per minute and then with respect to total protein production. This results in an overall accuracy of about $10 \%$. The result is a count of the differential rate of DnaK production (i.e., the fraction DnaK constitutes of total protein synthesis relative to the same fraction before the temperature shift [10]) as a function of time after the temperature shift. For the shift $T \rightarrow T+\Delta T$ at time $t=0$ we thus record

$$
r(T, t)=\frac{\text { Rate of DnaK production at time } t}{\text { Rate of DnaK production at time } t=0},
$$

where the denominator counts the steady state production of DnaK at temperature $T$. In Fig. 1 we display three examples, all associated with temperature changes of absolute magnitude $\Delta T=7{ }^{\circ} \mathrm{C}$. When changing $T$ from $30^{\circ} \mathrm{C}$ to $37^{\circ} \mathrm{C}$, one observes that $r$ increases to $\sim 6$ after a time of 0.07 generation. Later the expression rate relaxes to the normal level again, reflecting that other processes counteract the initial response. When reversing the jump, we see an opposite transient decrease in the expression rate. Finally, for $12^{\circ} \mathrm{C} \rightarrow 19^{\circ} \mathrm{C}$ we observe that the $T$ increase gives a decrease in the expression rate. Thus the cells' response to a positive temperature jump is opposite at low temperature $T$ than it is at high $T$.

Figure 2 summarizes our findings by plotting the value of $r=R$, where the deviation from $r=1$ is largest for a number of shifts $T \rightarrow T+7{ }^{\circ} \mathrm{C}$. $R$ is fitted by

$$
\ln [R(T)]=(\alpha \Delta T)\left(T-T_{0}\right),
$$




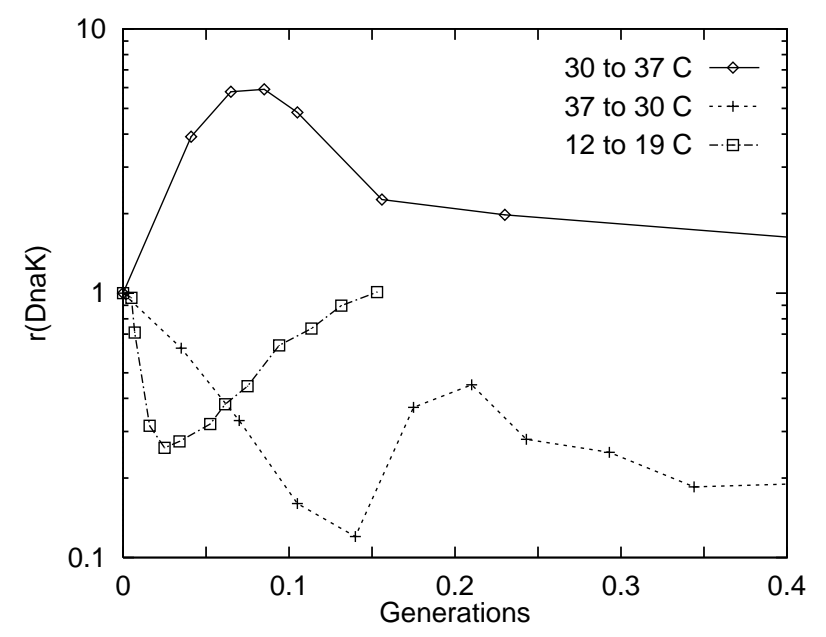

FIG. 1. Heat-shock response measured as DnaK rate production change as a function of time since a temperature shift. The production is normalized with an overall protein production rate, as well as with its initial rate. In all cases, we use absolute $\Delta T=7{ }^{\circ} \mathrm{C}$. The time scale is in units of one bacterial generation at the initial temperature.

where $R\left(T=T_{0}=23^{\circ} \mathrm{C}\right)=1$ and $\alpha \Delta T=\frac{\ln \left(R_{1} / R_{2}\right)}{T_{1}-T_{2}}=$ $0.2 K^{-1}$ (i.e., $\alpha=0.03 K^{-2}$ ).

To interpret this result we first assume that the production rate of DnaK is controlled by two factors, a slowly varying factor $C$ that depends on the composition of some other molecules in the cell and an instantaneous chemical reaction constant $K$. Thus at time $t$ after a shift in temperature the production of DnaK in the cell is

$$
\begin{aligned}
\frac{d[\mathrm{DnaK}]}{d t}(t, T \rightarrow T+\Delta T)= & C(t, T \rightarrow T+\Delta T) \\
& \times K(T+\Delta T),
\end{aligned}
$$

where the initial composition of molecules, $C(t=0, T \rightarrow$ $T+\Delta T$ ), equals their equilibrium number at the temperature we changed from, i.e., $=C_{\text {eq }}(T)$.

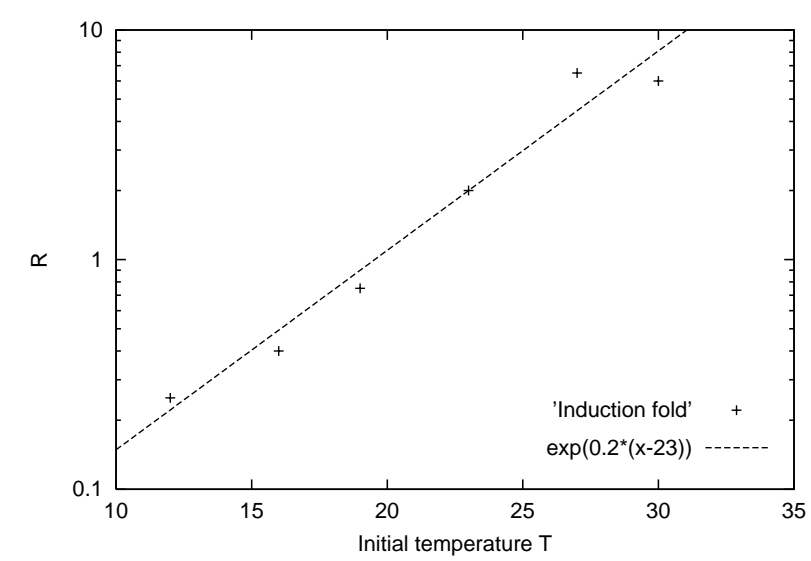

FIG. 2. Induction fold $R$ for positive temperature jumps as a function of initial temperature. The dashed line corresponds to the fit used in Eq. (2).
To the lowest approximation, where we even ignore feedback from changed DnaK in the cell until the DnaK production rate has reached its peak value,

$$
R=\frac{K(T+\Delta T)}{K(T)}
$$

which implies that

$$
\ln (R)=\ln [K(T+\Delta T)]-\ln [K(T)]=\frac{d \ln (K)}{d T} \Delta T .
$$

By using the linear approximation in Fig. 2,

$$
\ln (K) \approx \text { const }+\frac{\alpha}{2}\left(T-T_{0}\right)^{2} .
$$

Identifying $K=\exp (-\Delta G / T)$, the effective free energy associated with the reaction is

$$
\Delta G \approx G_{0}-\frac{\alpha T}{2}\left(T-T_{0}\right)^{2} .
$$

Thus $\Delta G$ has a maximum at $T=T_{0}=23^{\circ} \mathrm{C}$.

To interpret the fact that HS is connected to a $\Delta G$ that has a maximum at $T=T_{0} \approx 23{ }^{\circ} \mathrm{C}$, we note that many proteins exhibit a maximum stability at $T$ between $10^{\circ} \mathrm{C}$ and $30^{\circ} \mathrm{C}[11,12]$. Thus $\Delta G=G$ (folded) $-G$ (unfolded) connected to the folded state of a protein is at a minimum at $T_{0}$. The corresponding maximum of stability is the result of a balance between destabilization from entropy of polymer degrees of freedom at high $T$ and destabilization due to decreased entropic contribution to hydrophobicity at low $T$ $[12,13]$. One should also expect similar behavior for some parts of a protein [13], and thus expect a maximum binding for hydrophobic protein-protein associations at about $T_{0}$. Quantitatively, the size of the $\Delta G$ change inferred from the measured value of $\alpha=0.03 K^{-2}$ corresponds to a changed $G$ of about $20-30 \mathrm{kT}$ (about $15 \mathrm{kcal} / \mathrm{mol}$ ), for a temperature shift of about $40-50{ }^{\circ} \mathrm{C}$. This matches the change observed for typical single domain proteins [12]. Thus the HS is associated with a $\Delta G$ change equivalent to the destabilization of a typical protein.

The above picture still leaves us with the puzzle that protein binding and folding stability are at a maximum at about $T_{0}$, whereas the effective $\Delta G$ we observe has a minimum there. This can only be reconciled if the interaction we consider is inhibitory. An inhibitory binding that controls the feedback is indeed possible [3]. To summarize, in Fig. 3 we display the molecular network that we believe is controlling the transient heat-shock levels of DnaK in the cell. The key inhibitory control mechanism is the association of DnaK to $\sigma^{32}$. DnaK binds to unfolded protein residues [7], and the amount of DnaK- $\sigma^{32}$ association thereby monitors cellular consequences of a shift in temperature.

Impacts of mutants: We have measured the heat shock in a strain where the $\sigma^{32}$ gene is located on a high copy number plasmid. In this strain where the synthesis rate for $\sigma^{32}$ may approach that of DnaK, we found a HS that 


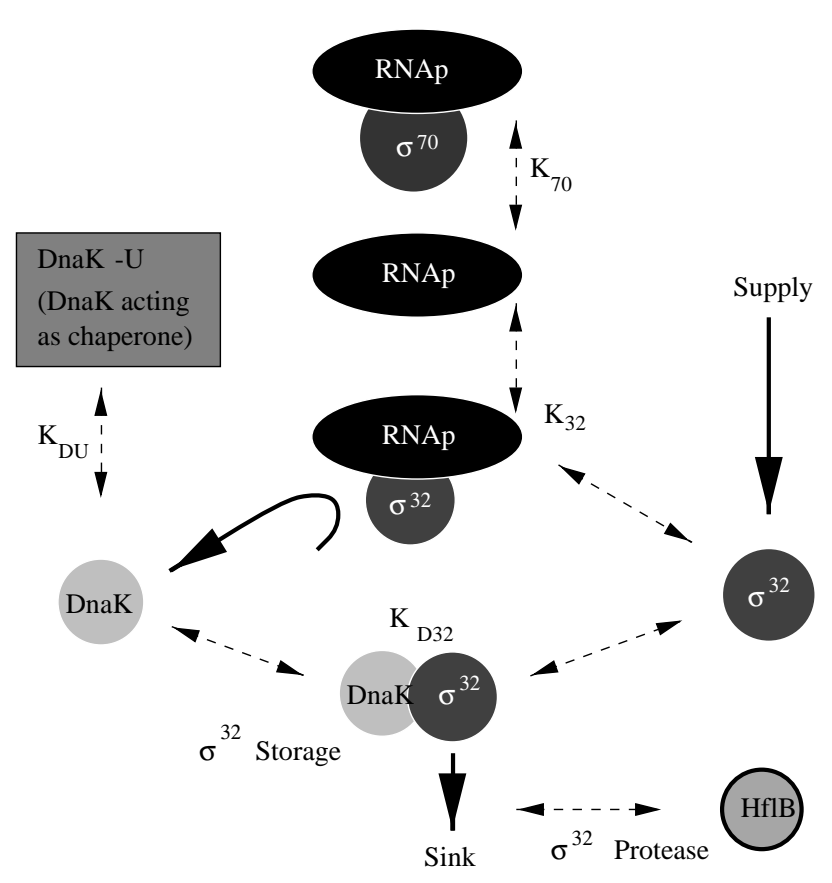

FIG. 3. Sufficient molecular network for the early heat shock. All dashed lines with arrows at both ends are chemical reactions which may reach equilibrium within a few seconds (they represent the homeostatic response). The solid directed arrows represent one-way reactions, with the production of DnaK through the $\sigma^{32}$-RNAp complex being the central one in this work. The time and temperature dependence of the early HS is reproduced when most DnaK is bound to unfolded proteins, and when remaining DnaK binds to $\sigma^{32}$ to facilitate a fast depletion of $\sigma^{32}$ through degradation by protease HflB.

was smaller and also remained positive down to temperature jumps from $T$ well below $T_{0}=23{ }^{\circ} \mathrm{C}$. According to Fig. 3 this reflects a situation where both $\sigma^{32}$ and DnaK are increased. The increased DnaK may then exceed the amount of unfolded proteins, and free DnaK concentration thus becomes nearly independent of the overall state of proteins in the cell. Further, an increase in the $\sigma^{32}$ supply decreases the possibility for the sink to act effectively. Thereby, other effects such as, e.g., the temperature dependence of the binding $\sigma^{32}$-RNAp versus the binding $\sigma^{70}$-RNAp (i.e., $K_{32} / K_{70}$ from Fig. 3 ), may govern the response.

The reaction network in Fig. 3 allows a more careful analysis of the production rate of DnaK:

$$
\frac{d[\mathrm{DnaK}]}{d t} \propto\left[\mathrm{RNAp}-\sigma^{32}\right] \approx \frac{\left[\sigma^{32}\right]}{1+g[\mathrm{DnaK}]},
$$

where the $\sigma^{32}$ changes when bound to DnaK due to degradation by proteases (the "sink" in Fig. 3):

$$
\begin{aligned}
\frac{d\left[\sigma_{32}\right]}{d t} & \propto \text { supply }-\left[\text { DnaK- } \sigma^{32}\right] \\
& \approx \text { supply }-\frac{\left[\sigma^{32}\right]}{1+(g[\text { DnaK }])^{-1}} .
\end{aligned}
$$

Here $g=\exp (-\Delta G / T)$ is an effective reaction constant. In the approximation where we ignore free $\sigma^{32}$, free $\sigma^{70}$, and the fraction of DnaK bound by $\sigma^{32}$, then

$$
g=\left(\frac{K_{70}\left[\sigma^{70}\right]}{K_{32}[\mathrm{RNAp}]}\right)\left(\frac{K_{D 32}}{1+K_{D U}\left[U_{f}\right]}\right) .
$$

The first term expresses the $\sigma$ 's competition for RNAp binding, whereas the second term expresses the DnaK controlled response. $\left[U_{f}\right]$, which denotes unfolded proteins that are not bound to DnaK, decreases with increasing [DnaK].

When moving away from $T_{0}$, i.e., lowering $g$ by increasing $\left[U_{f}\right]$, the rate for DnaK production increases. For an approximately unchanged "supply," the extremum in production occurs when $d\left[\sigma^{32}\right] / d t=0$ and has a value that is approximately $\propto 1 / g$. With the assumption that supply does not have time to change before extreme response is obtained, we identify $R$ with $1 / g$ and thereby with the free energy difference $\Delta G$ that controls the HS. The early rise in $r$ is reproduced when most $\sigma^{32}$ are bound to RNAp reflected in the condition $g[\mathrm{DnaK}] \ll 1$. This implies a significant increase in the $\sigma^{32}$ lifetime under a positive HS, and implies that the early HS is due to a changed depletion rate of $\sigma^{32}$. Later, the response is modified, partly by a changed supply and finally by a changed level of the heat-shock-induced protease HflB (HflB is a protease protein, which means that it actively degrades other proteins) that depends on and counteracts the $\sigma^{32}$ level in the cell.

The largest uncertainty in our analysis is the possibility of a significant time variation in supply and HflB levels during the HS. As these will govern the late stages of the heat shock, the variation in " $\Delta G$ " for proteins in the cell may easily be underestimated by using the peak height variation with $T$. Adding to the uncertainty in what $\Delta G$ precisely represents is also the fact that, although we only measure DnaK, it can be the complex of the heat-shock proteins DnaK, GrpE, and DnaJ (GrpE and DnaJ are chaperone proteins and as DnaK they are regulated by $\sigma^{32}$ ) which sense the state of unfolded proteins in the cell $[9,14]$. Such cooperativity may amplify the heat shock.

For the final interpretation of $\Delta G$ we stress that it effectively counts the free energy difference between the complex DnaK- $\sigma^{32}$ and that of DnaK being free or being bound to unfolded proteins in the cell. Dependent on the fraction of DnaK relative to unfolded proteins $[U]$ in the cell, i.e., whether $K_{D U}\left[U_{f}\right]$ is larger or smaller than 1, the HS may or may not depend on the overall folding stability of proteins in the cell. Thus for much more unfolded proteins than DnaK in the cell, the measured $\Delta G$ reflects both an increase of the binding to unfolded residues $K_{D U}\left[U_{f}\right]$ as well as a decrease of the DnaK- $\sigma^{32}$ binding $K_{D 32}$ when moving away from $T_{0}$. Our data do not discriminate between these processes. This discrimination can, however, be obtained in [15], where it was found that overexpression of DnaK through a $\sigma^{32}$ dependent pathway represses HS. As DnaK- $\sigma^{32}$ binding still plays a crucial role in this 
setup, the vanishing HS of [15] supports a scenario where too much DnaK imply $K_{D U}\left[U_{f}\right] \ll 1$. Then $g$ in Eq. (10), and thereby the $\sigma^{32}$ response, becomes insensitive to the amount of unfolded proteins in the cell.

We conclude that the HS is induced through the changed folding stability of proteins throughout the cell, sensed by a changed need of chaperones. This may reflect primarily an increased amount of proteins that is on the way to becoming folded, and not necessarily an increased denaturation of already folded proteins.

We now discuss related proposals of "cellular thermometers" for the HS. McCarthy and Walker [16] proposed that the thermometer was a change in autophosphorylation of the DnaK protein, giving it a temperature dependent activity. However, their data do not indicate that the reversed HS response that we observe at $T<23{ }^{\circ} \mathrm{C}$ could be caused by such a mechanism. Gross [3] made an extensive network of possible chemical feedback mechanisms which connect a rise in the $\sigma^{32}$ level with the folding state of proteins in the cell: an increased synthesis of $\sigma^{32}$, an increased release of $\sigma^{32}$ from DnaK, as well as an increased stability of $\sigma^{32}$ when DnaK gets bound to unfolded protein residues. Figure 3 specifies these possibilities to a minimalistic chemical response including the two latter mechanisms combined, and, of these, only the option of a changed stability of $\sigma^{32}$ due to a sink controlled by DnaK- $\sigma^{32}$ is able to also reproduce the fact that the maximum HS takes time to develop. In regard to the work by Morita et al. [17], which proposed an increased synthesis of $\sigma^{32}$, we note that, for high temperatures $T$, the major mechanism that controls $\sigma^{32}$ synthesis, in fact, is a $T$ dependent change in the mRNA structure that leads to an increased translation at increased $T$ [17]. However, again our finding of a reversed HS at $T<23{ }^{\circ} \mathrm{C}$ is not readily explained by such changes in the stability of mRNA structures below $23^{\circ} \mathrm{C}$.

In summary, we observed that positive heat shock is induced when $T$ changes from $T_{0} \sim 23{ }^{\circ} \mathrm{C}$. We found that the size of the heat shock qualitatively as well as quantitatively follows the thermodynamic stability of proteins with temperature. This suggested that stability related to hot as well as to cold unfolding of proteins is implemented in the HS. We demonstrated that such an implementation was possible in a minimalistic chemical network, where the control is through an inhibitory binding of the central heat-shock proteins. Finally, we saw that the temporal behavior of the HS is reproduced when this inhibitory binding controls the heat shock by exposing $\sigma^{32}$ to a protease.

[1] S. Lindquist, Annu. Rev. Biochem. 55, 1151 (1986).

[2] F. C. Neidhardt, R. A. VanBogelen, and V. Vaughn, Annu. Rev. Genet. 18, 295 (1984).

[3] C. A. Gross, Cellular and Molecular Biology (ASM Press, Washington, D.C., 1996), p. 1382.

[4] Y. Zhou, N. Kusukawa, J. W. Erickson, C. A. Gross, and T. Yura, J. Bacteriol. 170, 3640 (1988).

[5] S. L. Herendeen, R. A. VanBogelen, and F. C. Neidhardt, J. Bacteriol. 139, 185 (1979).

[6] S. Pedersen, P. L. Bloch, S. Reeh, and F. C. Neidhardt, Cell 14, 179 (1978).

[7] A. Gragarov, L. Zeng, X. Zhao, W. Burkholder, and M. E. Gottesman, J. Mol. Biol. 235, 848 (1994).

[8] D. B. Straus, W. A. Walter, and C. A. Gross, Nature (London) 329, 348 (1987).

[9] K. Tilly, J. Spence, and C. Georgopoulos, J. Bacteriol. 171, 1585 (1989).

[10] S. Reeh, S. Pedersen, and J. D. Friesen, Molec. Gen. Genet. 149, 279 (1976).

[11] P. L. Privalov, E. I. Tiktopulo, S. Yu. Venyaminov, Yu. V. Griko, G. I. Makhatadze, and N. N. Khechinashvili, J. Mol. Biol. 205, 737 (1989).

[12] G. M. Makhatadze and P. L. Privalov, Adv. Protein Chem. 47, 307 (1995).

[13] A. Hansen, M. H. Jensen, K. Sneppen, and G. Zocchi, Eur. Phys. J. B 6, 157 (1998).

[14] D. B. Straus, W. A. Walter, and C. A. Gross, Genes Dev. 4, 2202 (1990).

[15] K. Tilly, N. McKittrick, M. Zylicz, and C. Georgopoulos, Cell 34, 641 (1983).

[16] J. S. McCarthy and G. C. Walker, Proc. Natl. Acad. Sci. U.S.A. 88, 9513 (1991).

[17] M. T. Morita, Y. Tanaka, T.S. Kodama, Y. Kyogoku, H. Yanagi, and T. Yura, Genes Dev. 13, 655 (1999). 